\title{
RECHTSSCHUTZ VON INVESTITIONEN IN ENTWICKLUNGSLÄNDERN UND INVESTITIONSENTSCHEIDUNG
}

\author{
Von HeINRICH JÜTTNER
}

Die Forderungen der Entwicklungsländer nach einer neuen Weltwirtschaftsordnung, wie sie zuletzt auf der 4. Welthandelskonferenz der Vereinten Nationen im Mai 1976 in Nairobi zum Ausdruck kamen, nehmen auf allen internationalen Tagungen einen immer größeren Raum ein. Die Länder der Dritten Welt verfolgen dieses Thema mit um so größerer Beharrlichkeit, als sie in der gegenwärtigen Wirtschaftsordnung nur geringe Chancen sehen, ihren Rückstand gegenüber den Industriestaaten aufzuholen.

\section{Souveränität über ausländische Investitionen}

Eine der wichtigsten Forderungen, die in diesem Zusammenhang aufgestellt werden, ist die volle Souveränität eines jeden Staates über seine natürlichen Reichtümer und über alle wirtschaftlichen Aktivitäten in seinem Hoheitsgebiet bis hin zur Nationalisierung bzw. Enteignung. Schon in der im April 1974 auf der 6. Sondersitzung der Vereinten Nationen verabschiedeten „Deklaration über die Errichtung einer Neuen Weltwirtschaftsordnung" wird (unter Punkt 4e) dieses Souveränitätsprinzip betont. In Artikel 2 der am 12. Dezember 1974 von der 29. UN-Vollversammlung beschlossenen Charta der wirtschaftlichen Rechte und Pflichten der Staaten heißt es dann unmißverständlich:

"Jeder Staat hat die volle und ständige Souveränität einschließlich des Besitz-, des Nutzungs- und des Verfügungsrechts über alle seine Reichtümer, Naturschätze und wirtschaftlichen Betätigungen und übt diese Souveränität ungehindert aus.

Jeder Staat hat das Recht,

a) die Aufsicht über ausländische Investitionen in seinem nationalen Hoheitsbereich nach Maßgabe seiner Rechts- und sonstigen Vorschriften und entsprechend seinen nationalen Zielen und Prioritäten zu regeln und auszuüben. Ein Staat darf nicht gezwungen werden, für ausländische Investitionen Vorzugsbehandlungen zu gewähren; ...

c) ausländisches Vermögen $\mathrm{zu}$ verstaatlichen, $\mathrm{zu}$ enteignen oder das Eigentum daran zu übertragen; in diesem Fall soll der diese Maßnahmen treffende Staat unter Berücksichtigung seiner einschlägigen Rechts- und sonstigen Vorschriften und aller von ihm für wesentlich erachteten Umstände eine angemessene Entschädigung zahlen. Ist die Frage der Entschädigung im Einzelfall umstritten, so wird sie nach dem innerstaatlichen Recht des verstaatlichenden Staates und von seinen Gerichten beigelegt, sofern nicht alle betroffenen Staaten frei und einvernehmlich vereinbaren, auf der Grundlage der souveränen Gleichheit der Staaten und im Einklang mit dem Grundsatz der freien Wahl der Mittel eine andere friedliche Art der Beilegung zu suchen." 
Auch die von der zweiten Generalkonferenz der UNIDO (United Nations Industrial Development Organization) im März 1975 in Lima verabschiedete „Erklärung über industrielle Zusammenarbeit“ unterstreicht, „daß jeder Staat das unabdingbare Recht besitzt, frei seine Souveränität und ständige Kontrolle auszuüben, über seine natürlichen Ressourcen - auf dem Festland ebenso wie im Meer sowie über seine gesamte wirtschaftliche Aktivität zur zweckdienlichen Nutzung dieser Reserven einschließlich ihrer Nationalisierung in Übereinstimmung mit seinen Gesetzen als einem Ausdruck dieses Rechtes frei zu bestimmen, und daß kein Staat irgendwelchen wirtschaftlichen, politischen oder anderen Pressionen ausgesetzt werden darf, die ihn an der vollen und freien Wahrnehmung dieses unabänderlichen Rechtes hindern ${ }^{1 “}$.

Entscheidend und neu sind hierbei die Aussagen, daß die Enteignung und die Festlegung einer Entschädigung nach innerstaatlichem Recht erfolgen sollen. Hiermit wird eine bewußte Abkehr von den Regeln des klassischen Völkerrechts vollzogen. Diese lassen zwar Verstaatlichungen und Enteignungen durchaus zu, sie legen jedoch einen sogenannten „Mindeststandard“ zugrunde, der u. a. beinhaltet, daß Ausländer nicht gegenüber Einheimischen diskriminiert werden dürfen, daß Enteignungen nur im öffentlichen Interesse und nur aufgrund gesetzlicher oder rechtlicher Bestimmungen erfolgen dürfen und daß sie mit einer angemessenen, prompten und effektiv verwertbaren Entschädigung verbunden sein müssen.

Eine Substanzgarantie oder die Pflicht zum vollständigen Schadensersatz gibt es demnach im Völkerrecht nicht. Auch das Grundgesetz der Bundesrepublik Deutschland läßt bekanntlich in Artikel 14 eine Enteignung unter den genannten Bedingungen ausdrücklich $\mathrm{zu}$ und legt fest, daß die „Entschädigung . . . unter gerechter Abwägung der Interessen der Allgemeinheit und der Beteiligten zu bestimmen" ist.

Der aus den zitierten Deklarationen erkennbar werdende Widerspruch zwischen der Betonung der staatlichen Souveränität und Verfügungsmacht einerseits und dem rechtlichen Schutz ausländischen Eigentums andererseits läßt daher - vor allem im Zusammenhang mit den Problemen der Entwicklungshilfe - der Beurteilung des Investitionsklimas in Entwicklungsländern und der Absicherung von ausländischen Kapitalanlagen in diesen Gebieten besondere Bedeutung zukommen. Zudem befinden sich die Entwicklungsländer gegenüber den ausländischen Investitionen in einer zwiespältigen Lage. Einerseits brauchen sie zur Verfolgung ihrer wirtschaftlichen Ziele ausländisches Kapital und Know-how, andererseits aber befürchten sie eine Einmischung in innenpolitische Themen und eine Überfremdung der eigenen Wirtschaft. Ihre Politik muß daher immer darauf zielen, sowohl ein attraktives Investitionsklima einschließlich entsprechender Förderungsmaßnahmen zu bieten als auch durch geeignete Auflagen und Kontrollmaßnahmen einen möglichst großen Nutzen aus den Direktinvestitionen zu ziehen.

Daher stehen die Entwicklungsländer den ausländischen Kapitalanlagen keineswegs grundsätzlich ablehnend gegenüber. Dies kommt auch in internationalen Resolutionen immer wieder zum Ausdruck; so heißt es z. B. unter Punkt IV. 6. der Entschließung der 7. Sonder-Generalversammlung der Vereinten Nationen: „Die entwickelten Länder sollten wann immer möglich ihre Unternehmen ermutigen,

1 Deutsche Ubersetzung beider Dokumente in: Entwicklungspolitik-Materialien Nr. 49. Bundesministerium für wirtschaftliche Zusammenarbeit, Bonn, Juni 1975. 
sich im Rahmen der Entwicklungspläne und -programme der Entwicklungsländer, die dies wünschen, an Investitionsvorhaben zu beteiligen; diese Beteiligung sollte gemäß den Rechtsvorschriften der betreffenden Entwicklungsländer erfolgen."

Allerdings erlaubt die Tatsache, daß ein wesentlicher, den wirtschaftlichen Fortschritt der Entwicklungsländer hemmender Faktor ihr Mangel an Investitionskapital ist und daß ausländische Kapitalanlagen diesen Bedarf zum Teil stillen, keine Aussage darüber, ob Direktinvestitionen als Entwicklungshilfe zu bezeichnen sind. Zudem sehen die Entwicklungsländer die Bedeutung der Direktinvestitionen keineswegs allein in der Kapitalzufuhr, sondern auch in ihren Nebenwirkungen wie der Übertragung von technischem und wirtschaftlichem Know-how, der Stimulierung eines einheimischen Unternehmertums, der Ausbildung und Beschäftigung von Arbeitskräften oder der Devisenersparnis oder -erwirtschaftung.

\section{Deutsche Direktinvestitionen in Entwicklungsländern}

Der Gesamtbestand der deutschen Direktinvestitionen - direkt im Rahmen unternehmerischer Betätigung erworbene Eigentumsrechte im Ausland - betrug Ende 1975 nach den Angaben des Bundesministers für Wirtschaft fast $42 \mathrm{Mrd}$. DM. von denen etwa $30 \%$ in Entwicklungsländern lagen. Dieser Prozentsatz ist in den letzten zehn Jahren nahezu unverändert geblieben (Einzelheiten zeigt Tabelle 1). Mehr als zwei Drittel der deutschen außereuropäischen Investitionen in Entwicklungsländern liegen in Lateinamerika, obwohl dort nur sieben Prozent der Weltbevölkerung leben, zwanzig Prozent sind in Asien - mit sechzig Prozent der Bevölkerung - und zehn Prozent in Afrika.

Sechzig Prozent der deutschen Investitionen in Entwicklungsländern entfallen auf Chemie- und Kunststoffindustrie, Elektrotechnik, Feinmechanik, Maschinenbau, Fahrzeugbau und Schiffbau, während die Bereiche Erdölgewinnung und -verarbeitung sowie Bergbau nur knapp sieben Prozent ausmachen. Bei den US-amerikanischen, den britischen und den französischen Direktinvestitionen in Entwicklungsländern ist jeweils etwa die Hälfte auf den Rohstoffbereich konzentriert, in dem - wie bereits die Formulierungen in den erwähnten Resolutionen zeigen - Verstaatlichungen am ehesten zu erwarten und in der Vergangenheit auch erfolgt sind.

Vom Standpunkt der Bundesrepublik Deutschland aus erscheinen deutsche Direktinvestitionen in Entwicklungsländern aufgrund verschiedener Überlegungen begrüßenswert. Zunächst ist da einmal das quantitative Moment: Ohne Einbeziehung der privaten Kapitalleistungen (Direktinvestitionen, Wertpapieranlagen, Exportkredite), die in den letzten Jahren stets etwa ebenso umfangreich waren wie die öffentlichen Leistungen, könnte die Bundesrepublik ihrer 1968 auf der zweiten Welthandelskonferenz eingegangenen Zusage, jährlich einen Nettobetrag von mindestens einem Prozent ihres Bruttosozialprodukts den Entwicklungsländern finanziell zur Verfügung zu stellen, auch nicht entfernt nachkommen. Der Anteil öffentlicher Leistungen schwankt seit 1970 zwischen 0,3 und 0,4 Prozent, insgesamt wurden etwa 0,5 bis 0,9 Prozent erreicht. Die öffentliche Hand war also schon rein quantitativ nicht in der Lage, den Kapitalbedarf der Entwicklungsländer zu befriedigen. 
Tabelle 1:

Entwicklung der deutschen Direktinvestitionen in Industrie- und Entwicklungslän

$\mathrm{Jahr}$

$\begin{array}{lccc}\text { Netto- } & \text { Stand am } & \text { Netto- } & \text { Stand am } \\ \text { zugang } & \text { Jahresende } & \text { zugang } & \text { Jahresende }\end{array}$

\begin{tabular}{crrrrr}
\hline $1952-1955$ & 257,6 & 257,6 & 163,5 & 163,5 & \\
1956 & 293,3 & 550,9 & 116,6 & 280,1 \\
1957 & 329,6 & 880,5 & 188,6 & 468,7 \\
1958 & 317,8 & 1198,3 & 191,6 & 660,3 \\
1959 & 339,9 & 1538,2 & 223,7 & 884,0 & \\
1960 & 458,0 & 1996,2 & 281,6 & 1165,6 & \\
1961 & 441,0 & 2437,2 & 239,7 & 1405,3 & \\
1962 & 690,8 & 3128,0 & 422,4 & 1827,7 & 1 \\
1963 & 840,9 & 3968,9 & 274,2 & 2101,9 & 1 \\
1964 & 945,4 & 4914,3 & 188,9 & 2290,8 & 1 \\
1965 & 856,6 & 5770,9 & 255,4 & 2546,2 & 1 \\
1966 & 1187,8 & 6958,7 & 490,4 & 3036,6 & 1 \\
1967 & 1626,1 & 8584,8 & 435,4 & 3472,0 & 2 \\
1968 & 1573,8 & 10158,6 & 718,4 & 4190,4 & 2 \\
1969 & 2076,3 & 12234,9 & 1193,0 & 5383,4 & 3 \\
1970 & 2665,8 & 14900,7 & 828,9 & 6212,3 & 3 \\
1971 & 2201,5 & 17102,2 & 465,9 & 6678,2 & 2 \\
1972 & 1617,0 & 18719,2 & 1198,7 & 7876,9 & 2 \\
1973 & 3863,7 & 22582,9 & 1772,8 & 9649,7 & 5 \\
1974 & 3376,8 & 25959,7 & 1150,6 & 10800,3 & 4 \\
1975 & 3743,9 & 29703,6 & 1481,2 & 12281,5 & 5 \\
\hline
\end{tabular}

Quelle : Zusammengestellt nach den Runderlassen Außenwirtschaft des Bundesi Tages-Buchwerte der deutschen Direktinvestitionen liegen nach Schätzungen um 20 
Weitere Argumente zugunsten der deutschen Direktinvestitionen in Entwicklungsländern sind die Beseitigung bzw. Verminderung des Handelsbilanzüberschusses, der Abbau der Exportlastigkeit der deutschen Wirtschaft, die Sicherung der Rohstoffversorgung, die Gewinnung ausländischer Märkte und entwicklungspolitische Uberlegungen. In der „Entwicklungspolitischen Konzeption der BRD“ (Neufassung 1975, Punkt 16) heißt es dazu: „Die Bundesregierung erkennt die große Bedeutung des privaten Kapitaltransfers in Entwicklungsländer an. Deren eigene Aufgabe ist es, ausländische Investitionen nach ihren eigenen Zielen und Prioritäten zu lenken und zu fördern. Die Bundesregierung begrüßt ihrerseits besonders private Direktinvestitionen, die partnerschaftlich angelegt sind und zum Beispiel durch technologische Ausstrahlung, Nutzung und Entfaltung heimischer Ressourcen, Ergänzung einheimischer Produktionsstrukturen, Exportdiversifizierung und Beschäftigung in besonderem Maße den wirtschaftlichen und gesellschaftlichen Bedürfnissen der Entwicklungsländer dienen."

Aus diesen verschiedenartigen Uberlegungen heraus sieht sich die Bundesrepublik auch veranlaßt, deutsche Direktinvestitionen in Entwicklungsländern zu fördern und zu schützen. Auch die entwicklungspolitische Konzeption betont daher, daß die Bundesregierung "die Notwendigkeit der Rechtsicherheit und eines gesunden Investitionsklimas in den Entwicklungsländern unterstreichen" wird.

\section{Möglichkeiten der rechtlichen Absicherung}

$\mathrm{Da}$ also Industrie- und Entwicklungsländer gleichermaßen - wenn auch aus unterschiedlichen Beweggründen — am Zufluß ausländischen Privatkapitals in die Entwicklungsländer interessiert sind, haben sie ein vielfältiges System von staatlichen Förderungsmaßnahmen für Auslandsinvestitionen aufgebaut, die deren Risiko mindern oder die Finanzierung erleichtern sollen.

Nach den „Initiatoren“ lassen sich drei Gruppen investitionsfördernder Einrichtungen unterscheiden:

- Maßnahmen allein des kapitalexportierenden Staates (z. B. Garantien, Steuervorteile, Finanzierungshilfen),

- Maßnahmen allein des kapitalimportierenden Landes (z. B. Steuererleichterungen oder -erlasse, Eigentumsgarantien, Niederlassungsverträge),

- Maßnahmen, die von kapitalexportierenden und kapitalimportierenden Staaten gemeinsam erfolgen (z. B. bilaterale Investitionsförderungsverträge oder internationale Abkommen wie die Weltbankkonvention).

Vom Ansatzpunkt der Förderung her werden im allgemeinen folgende vier Arten von Maßnahmen angeführt:

- Risikomindernde Maßnahmen (Garantien, Schutzabkommen, Schiedsgerichtsverträge),

- Steuerpolitische Maßnahmen (Steuererleichterungen oder -befreiungen),

- Finanzierungshilfen (Kredite und Beteiligungen, Zinssubventionen),

- Sonstige Maßnahmen (Devisenpolitische Regelungen, Informationshilfen, Ausbildungsförderung, länderkundliche und fremdsprachliche Vorbereitung).

In dem hier behandelten Zusammenhang interessieren vor allem die Bestrebungen, einen Ausgleich bzw. eine Absicherung für die besonderen mit einer Investition in einem Entwicklungsland verbundenen Risiken zu schaffen. In diese Richtung zielen die staatlichen Garantiesysteme, die bilateralen Investitionsschutzklauseln oder -verträge sowie die entsprechenden multilateralen Maßnahmen. 
Nahezu sämtliche westliche Industriestaaten kennen ein Versicherungs- oder Garantiesystem für Auslandsinvestitionen, wobei in der Regel Enteignungs-, Kriegsund Transferrisiken gedeckt werden, teils in allen Staaten, teils in Entwicklungsländern und teils nur in bestimmten, z. B. durch einen völkerrechtlichen Vertrag verbundenen Ländern. Die jährliche Versicherungsprämie bewegt sich je nach versichertem Risiko und Objekt (Anfangsinvestition, Reinvestition, Gewinn) zwischen $0,25 \%$ und $0,8 \%$, wobei die Garantiedauer zumeist 15 Jahre beträgt. Bei Verlusten ist im allgemeinen eine Eigenbeteiligung des Investors - im Durchschnitt $10 \%$ - erforderlich.

Eine andere Maßnahme zur Risikominderung sind bilaterale Kapitalschutzvereinbarungen, die zwischen Industrie- und Entwicklungsländern abgeschlossen werden und den Investoren verschiedene Sicherheiten geben sollen, z. B. gegen diskriminierende Gesetzes- oder Verwaltungsakte. Sie gewähren keinen Schutz vor Enteignungen, garantieren jedoch in solchen Fällen eine angemessene Entschädigung. Zum Teil enthalten diese Abkommen eine Reihe detaillierter Regelungen (wie bei der BRD oder Schweiz), zum Teil sind es nur allgemeine Investitionsschutzklauseln in Freundschafts- oder Handelsverträgen (wie bei den USA oder bei Frankreich).

Außer diesen Staaten haben auch Dänemark, Großbritannien, Italien, Japan, die Niederlande, Norwegen und Schweden Investitionsschutzabkommen oder -klauseln vereinbart. Die Bedeutung all dieser bilateralen Vereinbarungen ist einerseits darin zu sehen, daß sie Vertrauen in die rechtliche Stabilität des Entwicklungslandes hervorrufen und den ausländischen Unternehmen ein attraktives Investitionsklima anzeigen sollen. Andererseits liefern sie einen Beitrag zur Bildung und Festigung eines völkerrechtlichen Standards, insbesondere, wenn sie in größerer Zahl und mit übereinstimmendem Inhalt abgeschlossen werden.

Ein bislang nicht realisierter Vorschlag im internationalen Bereich ist die Idee eines multilateralen Garantiesystems für Direktinvestitionen, in das Industriewie Entwicklungsländer einbezogen werden sollen. Dadurch soll einerseits eine größere Risikostreuung erreicht werden, andererseits sollen die beteiligten Entwicklungsländer von entschädigungslosen Enteignungen ausländischen Kapitals abgehalten werden. Hierzu liegen Entwürfe aus verschiedenen Quellen vor, einer der wichtigsten ist der Plan einer „Multilateral Investment Insurance“ der Weltbank.

Die Zahl der für eine multilaterale Vermögens- oder Investitions-Schutzkonvention vorgelegten Pläne ist bedeutend größer, da sich bereits seit 1890 Staatenkonferenzen, internationale Organisationen und Verbände, wissenschaftliche Institute und Völkerrechtler mit dieser Frage befassen. Der bekannteste Vorschlag stammt von der OECD, die 1967 eine "Convention on the Protection of Foreign Property" vorlegte. Alle Bestrebungen um einen multilateralen Vermögensschutz scheiterten bislang jedoch an den divergierenden Ansichten der einzelnen Staaten. Diese vergeblichen Bemühungen um ein multilaterales Investitionsschutzabkommen haben dazu geführt, daß verstärkt in bilateralen Staatsverträgen der Schutz von Auslandsinvestitionen vereinbart wurde.

Dagegen sind die Bestrebungen um eine internationale Schiedsgerichtsbarkeit erfolgreich gewesen. Nach verschiedenen Vorarbeiten legte die Weltbank Anfang 1965 eine "Convention on the Settlement of Investment Disputes between States and Nationals of other States" vor, die im Herbst 1966 in Kraft trat. Bis Mitte 1975 hatten 66 Staaten dieses Abkommen ratifiziert und damit für eventuelle 
Streitigkeiten mit ausländischen Investoren die Zuständigkeit des bei der Weltbank eingerichteten Schiedszentrums vorgesehen. Die Ostblockstaaten und die lateinamerikanischen Ländern sind der Konvention allerdings nicht beigetreten.

Ergänzend ist darauf hinzuweisen, daß dem Empfängerland in jedem ausländischen Investor auch in begrenztem Rahmen die politische und wirtschaftliche Macht dessen Heimatstaates entgegentritt ${ }^{2}$. Dieser Machtfaktor ist zum Beispiel besonders deutlich im Falle der USA; er fällt um so weniger ins Gewicht, je geringer die wirtschaftliche und politische Macht des betreffenden Staates jeweils ist.

$\mathrm{Zu}$ erwähnen ist zunächst der diplomatische Dienst des Heimatstaates, dessen Rolle von direkter Einflußnahme auf Regierung und Regierungsbildung bzw. auf Parlament und Politiker bis hinunter zu Anregungen und Hinweisen auf den verschiedensten Ebenen reichen kann. Weiterhin kommt heute dem umfangreichen Instrumentarium der Entwicklungshilfe, ihrem Volumen und ihrer Kontinuität, einige Bedeutung zu. Ferner ist die Gewährung bzw. Verweigerung staatlicher Exportgarantien oder -kredite anzuführen. Außerdem spielt der Zugang oder Nichtzugang $z u$ westlichen Kapitalmärkten eine Rolle, der durch die Regierungen maßgeblich beeinflußt werden kann. Auch Weltbank und Internationaler Währungsfonds hängen bei ihrer Kapitalbeschaffung vom guten Willen finanzstarker Staaten ab. Schließlich steht auch noch das Mittel handelspolitischer Diskriminierung zur Verfügung.

\section{Garantien der Bundesrepublik Deutschland}

Deutsche Direktinvestitionen in Entwicklungsländern können - neben Eigentumsund Transfergarantien des Anlagelandes - durch staatliche deutsche Garantien, durch Investitionsförderungsvertäge und durch die Weltbank-Schiedsgerichtskonvention geschützt werden. Die „Bundesgarantien für Kapitalanlagen im Ausland“, eingeführt im Jahre 1958, können übernommen werden „zur Absicherung des politischen Risikos bei förderungswürdigen Kapitalanlagen im Ausland, wenn zwischen der Bundesrepublik und dem Land, in dem das Kapital angelegt wird, eine Vereinbarung über die Behandlung von Kapitalanlagen besteht oder, solange dies nicht der Fall ist, durch die Rechtsordnung des betreffenden Landes oder in sonstiger Weise ein ausreichender Schutz der Kapitalanlage gewährleistet erschein $\mathbf{t}^{3 \text { " }}$.

Die Garantieübernahme ist zwar nicht auf Entwicklungsländer beschränkt, wird aber praktisch so gehandhabt. Garantiefähige Kapitalanlagen sind Kapitalausstattungen von Niederlassungen oder Betriebsstätten deutscher Unternehmen im Ausland, Beteiligungen an ausländischen Gesellschaften und beteiligungsähnliche Darlehen sowie Erträge aus solchen Beteiligungen und Darlehen. Auf Antrag können auch reinvestierte Gewinne bis zur Höhe von 50\% des Einbringungskapitals in die Deckung einbezogen werden. Die Garantien gelten nur für künftige Investitionen. Ein Rechtsanspruch auf eine Garantiegewährung besteht nicht ${ }^{4}$.

Die Deckung gilt nur bei politischen Risiken, wirtschaftliche Risiken werden in die Bundesgarantien nicht einbezogen:

2 Vgl. Hans Bachmann: Die Problematik westlicher Privatinvestitionen in Entwicklungsländern. In: Die Rolle transnationaler Investitionen und multinationaler Gesellschaften in der modernen Weltwirtschaft. Zürich und St. Gallen 1973, S. 154-178, hier S. 167.

3 Haushaltsgesetz 1971, 99 Abs. 1 Nr. 3.

$4 \mathrm{Im}$ folgenden wird auf ein Merkblatt der Deutschen Revisions- und Treuhand-AG Treuarbeit und auf die Allgemeinen Bedingungen für die Übernahme von Garantien für Kapitalanlagen im Ausland vom Januar 1967 zurückgegriffen. 
- Verstaatlichung, Enteignung, enteignungsgleiche Eingriffe oder rechtswidrige Unterlassungen von Hoher Hand, die in ihren Auswirkungen einer Enteignung gleichkommen;

- Krieg, sonstige bewaffnete Auseinandersetzungen, Revolution oder Aufruhr;

- Zahlungsverbote, Moratorien;

- Unmöglichkeit der Konvertierung oder des Transfers.

Voraussetzung für die Übernahme einer Garantie ist, daß ein ausreichender Rechtsschutz der Kapitalanlage im Entwicklungsland gegeben ist. Dies wird z. B. beim Bestehen eines Investitionsförderungsvertrags angenommen, sofern er auf die Kapitalanlage Anwendung findet. Liegt ein solcher Vertrag nicht vor, so wird geprüft, ob ein ausreichender Schutz der Kapitalanlage durch die Rechtsordnung des betreffenden Landes oder in sonstiger Weise, z. B. durch den Beitritt dieses Landes zur Weltbank- Schiedsgerichts-Konvention, gegeben ist.

Außerdem müssen die Kapitalanlagen „förderungswürdig sein und sollen vorzugsweise zur Vertiefung der Beziehungen der Bundesrepublik Deutschland zu Entwicklungsländern beitragen“. So sollen die Garantien „in erster Linie übernommen werden für Kapitalanlagen bei solchen Unternehmen im Ausland, die sich mit der Erzeugung, der Gewinnung oder dem Vertrieb von Gütern oder mit Beförderungsleistungen befassen".

Die Laufzeit der Garantien beträgt bis zu 15 Jahre, im Ausnahmefall auch 20 Jahre. Für die Bearbeitung des Garantieantrags wird eine einmalige Gebür erhoben, die bei einem versicherten Betrag bis zu 10 Mill. DM 0,1\% und für den übersteigenden Betrag 0, 05\% \%, insgesamt jedoch höchstens $20000 \mathrm{DM}$ beträgt. Zu Beginn eines jeden Jahres ist ein laufendes Entgeld von 0,8\% vom jeweils garantierten Betrag zu zahlen.

Im Schadensfall leistet der Bund Ersatz für die durch politische Risiken eingetretenen Verluste. Dabei wird vom Zeitwert der Anlage ausgegangen, wobei gewisse Höchstbetragsregelungen gelten. So ist der Einbringungswert stets von grundlegender Bedeutung, und bei Erträgen erstreckt sich die Deckung auf höchstens $24 \%$ des in der Garantieerklärung festgehaltenen maximalen Deckungsumfangs. An dem ermittelten Verlust muß sich der Investor mit mindestens $5 \%$ selbst beteiligen.

Die Verluste werden nicht gedeckt, wenn sie auf einem Verhalten beruhen, das von dem Investor $\mathrm{zu}$ vertreten ist, oder wenn sie infolge von Ereignissen oder Maßnahmen auftreten, die auf einer ausländischen Beteiligung oder auf ausländischem Einfluß beim Garantienehmer beruhen. Dies wird angenommen, sofern sich Kapital oder Stimmrechte des Garantienehmers zu mehr als 50\% in ausländischer Hand befinden oder der Garantienehmer ausländischer Kontrolle unterliegt.

Neben der Garantie für Kapitalanlagen kann die Bundesregierung auch für an Lieferungen und Leistungen gebundene und für ungebundene Finanzkredite an ausländische Firmen bürgen. Diese Garantien schließen auch das wirtschaftliche Risiko ein, sind dafür aber teurer als Kapitalanlagegarantien. Für Direktinvestitionen in Entwicklungsländern sind die Garantien für Finanzkredite dann als Förderinstrument von Bedeutung, wenn Darlehen an Unternehmen mit einer Minderheitsbeteiligung gegeben werden oder wenn bei einem Darlehen, das ohne Einschaltung der Muttergesellschaft an ein ausländisches Tochterunternehmen gewährt wird, der Kreditgeber eine solche Garantie verlangt. 


\section{Deutsche Investitionsförderungsverträge}

Die Investitionsförderungsverträge der Bundesrepublik Deutschland mit Entwicklungsländern sind ein Mittel, das Kapitalanlagen in Entwicklungsländern einen möglichst großen Schutz vor Enteignungen gewähren soll. Im Gegensatz z. B. zu den USA hat die BRD dabei ein völlig neues Instrument zur Förderung und zum Schutz von privaten Auslandsinvestitionen eingesetzt. Der deutsche Vertragstyp beschäftigt sich allein mit dem Vermögensschutz, auch wenn er in verschiedenen Fällen mit Abkommen über die Gewährung technischer und wirtschaftlicher Hilfe verbunden wurde. Die Investitionsförderungsverträge regeln insbesondere die Inländerbehandlung und Meistbegünstigung, die Enteignung von Kapitalanlagen und die zu gewährende Entschädigung, den Transfer von Kapital, Entschädigung, Erträgen und Erlösen sowie die Einsetzung eines bilateralen Schiedsgerichtes. Außerdem dienen die Verträge - wie erwähnt - als Rechtsgrundlage für die Bundesgarantien für Kapitalanlagen im Ausland.

Bis März 1976 hatte die deutsche Bundesregierung 45 Investitionsförderungsverträge abgeschlossen, von denen 37 in Kraft sind Mit weiteren 15 Staaten wird mit unterschiedlicher Aussicht auf Erfolg verhandelt. Investitions- oder Eigentumsschutzklauseln gibt es auch in den Niederlassungs- sowie Freundschafts-, Handelsund Schiffahrtsverträgen, die mit sechs Entwicklungsländern bestehen.

Die Investitionsförderungsverträge werden in der Form eines Staatsvertrages geschlossen. Zunächst wird der Vertragsinhalt ausgehandelt und nach erfolgter Einigung über die einzelnen Bestimmungen paraphiert, d. h. abgezeichnet. Anschließend wird er von den beiden Staaten unterzeichnet - zumeist gleichzeitig - und den gesetzgebenden Organen zur Ratifizierung zugeleitet. Einen Monat nach dem Austausch der Ratifikationsurkunden tritt der Vertrag in Kraft.

Die Bezeichnung der deutschen Verträge ist trotz weitgehend identischem Inhalt unterschiedlich. So heißen sie Vertrag „über die Förderung von Kapitalanlagen“, „über die Förderung der Anlage von Kapital“, „zur Förderung und zum Schutz von Kapitalanlagen“. Dabei ist die letztgenannte Bezeichnung sowohl die meistbenutzte wie auch zutreffendste, da Förderung und Schutz nicht getrennt gesehen werden können und da auch das Prinzip der gegenseitigen Verpflichtung zum Ausdruck kommt, Kapitalanlagen aus dem anderen Staat vertragsgemäß zu behandeln.

Ausgangspunkt der Verhandlungen der deutschen Bundesregierung über den Abschluß eines Investitionsförderungsvertrages ist ein Mustervertrag, der einen möglichst umfassenden Schutz der deutschen Auslandsinvestitionen vorsieht. Zwischen dieser größtmöglichen Sicherung und einer völligen Schutzlosigkeit sind sämtliche Zwischenstufen denkbar, zumal die Tendenz, Bestimmungen des deutschen Musterentwurfs als unannehmbar abzulehnen, in den einzelnen Entwicklungsländern unterschiedlich ausgeprägt ist Andererseits wird es auch von der jeweiligen politischen Lage abhängen, inwieweit man auf die Einhaltung der vertraglichen Zusagen vertrauen darf, gleichgültig ob sie dem Musterentwurf folgen oder dahinter zurückbleiben. Die Bundesrepublik Deutschland ist grundsätzlich nur in Einzelbereichen zur Abweichung vom Mustervertrag bereit.

Die deutschen Investitionsförderungsverträge bestehen formell zumeist aus dem eigentlichen Vertrag selbst und aus einem Protokoll, das ebenfalls ratifiziert wird und dann ebenso verbindlich ist wie der Vertrag. Das Protokoll enthält Er- 
gänzungen, Auslegungsregelungen und Ausnahmen, Genehmigungs- oder Registrierungsverfahren sowie gegebenenfalls einseitige Verpflichtungen eines Vertragsstaates.

Der Inhalt des Mustervertrages, auf den hier kurz eingegangen wird, hat sich im Laufe der Jahre mehrfach geändert; die letzte Fassung stammt vom 1. Juli 1974. Den einzelnen Artikeln vorangestellt ist eine Präambel, die die Ausgangsbedingungen und Absichtserklärungen der Regierungen enthält. Sie kann gegebenenfalls zur Auslegung des Vertrages herangezogen werden.

Der Vertrag beginnt mit Definitionen der Begriffe „Kapitalanlagen“, „Erträge“, "Staatsangehörige" und "Gesellschaften“. Er steckt somit den Anwendungsbereich der materiellen Vertragsbestimmungen ab und macht den Schutzumfang des Vertrages überschaubar. Dabei ist zu erwähnen, daß der Begriff „Kapitalanlagen“ sehr weit gefaßt ist, da er „Vermögenswerte jeder Art" umschließt.

Die folgenden Artikel bringen dann die sogenannte "Wohlverhaltensklausel“, die eine gerechte und billige Behandlung der Investitionen aus dem Partnerstaat zusichert, sowie die Zusagen der Inländergleichbehandlung und der Meistbegünstigung, also Sicherheiten gegen eine Diskriminierung von Ausländern allgemein oder spezieller Nationalität.

Artikel 4 enthält das Kernstück des Vertrages, nämlich die Garantie, daß Enteignungen nur zum allgemeinen Wohl und gegen eine wertentsprechende, tatsächlich verwertbare, frei transferierbare und unverzüglich zu leistende Entschädigung erfolgen dürfen und daß die Rechtmäßigkeit der Enteignung und die Höhe der Entschädigung in einem ordentlichen Rechtsverfahren nachprüfbar sein müssen. Auch Maßnahmen, „die in ihren Auswirkungen einer Enteignung oder Verstaatlichung gleichkommen", - also schleichende Enteignung - sind in den Vertragsschutz einbezogen.

Zwei weitere Artikel sichern dem Investor den freien Transfer der im Zusammenhang mit der Kapitalanlage stehenden Zahlungen zu, insbesondere des Kapitals, der Erträge, eventueller Darlehen, von Lizenz- und anderen Gebühren sowie eines Liquidationserlöses. Weiterhin werden die Fristen festgelegt, innerhalb derer die Transferierungen - auch von Entschädigungszahlungen - zu erfolgen haben, und es wird unter Bezugnahme auf den Weltwährungsfonds der Wechselkurs bestimmt, der dabei anzuwenden ist.

Weiterhin enthält der Vertrag die sogenannte „Subrogationsklausel“. Sie besagt, daß ein Staat, der einen Investor aufgrund von Kapitalanlagegarantien für einen Verlust entschädigt hat, in die Rechte des Investors eintritt und diese gegenüber dem Anlageland - das den Rechtübergang anerkennen muß - geltend machen kann. In einem anderen Artikel findet sich die „Besserungsklausel“, wonach anderweitige innerstaatliche oder völkerrechtliche Regelungen, die den Investor günstiger stellen, den Bestimmungen des Investitionsförderungsvertrages vorgehen. Eine "Abschirmungsklausel“ zieht darüber hinaus etwaige Sondervereinbarungen zwischen Investor und Gastland in den völkerrechtlichen Schutz des Vertrages mit ein. Mit einem weiteren Artikel bemüht sich die Bundesregierung, auch jene Kapitalanlagen in den vertraglichen Schutz einzuschließen, die beim Abschluß des Vertrages bereits bestehen.

Artikel 10 bestimmt, daß Investitionsstreitigkeiten, sofern sie nicht zwischen den Regierungen der Vertragsparteien auf Konsultationsebene beigelegt werden, einem Schiedsgericht unterbreitet werden können. Weiterhin regelt er die Bildung und $\mathrm{Zu}-$ 
sammensetzung des Schiedsgerichtes und die Verfahrensregeln. Die Entscheidungen dieses Schiedsgerichtes sind bindend.

Im Gegensatz zu dem multilateralen Weltbank-Übereinkommen zur Beilegung von Investitionsstreitigkeiten hat hier der Investor keine Möglichkeit, ein Schiedsgerichtsverfahren einzuleiten. Parteien sind nur die beiden Vertragsstaaten. Allerdings kann das vertragliche Schiedsgericht insoweit nicht angerufen werden, „als zwischen dem Staatsangehörigen oder der Gesellschaft einer Vertragspartei und der anderen Vertragspartei eine Vereinbarung nach Maßgabe des Artikels 25 des (Weltbank-) Übereinkommens zustande gekommen ist.“

Eine Sachentscheidung des Schiedsgerichtes nach dem Investitionsförderungsvertrag kann nach allgemeinem Völkerrecht erst dann angestrebt werden und ergehen, wenn der Investor - oder bei Rechtsnachfolge der Staat - den innerstaatlichen Rechtsweg ausgeschöpft hat. Die Frage der Vollstreckung der vom Schiedsgericht ergangenen Entscheidung ist im Vertrag nicht geregelt. Da keine übergeordnete Instanz eingeschaltet ist, stößt man hier an die Grenzen der Souveränität der Vertragspartner.

Die Schlußartikel sichern den vertraglichen Schutz - in eingeschränktem Umfang - auch für den Fall kriegerischer oder ähnlicher Auseinandersetzungen zwischen den Vertragsparteien zu, beziehen das Land Berlin mit ein und regeln das Inkrafttreten des Vertrages, seine Laufzeit und seine Kündigung. Ziel der Bundesregierung ist es, eine Gültigkeitsdauer von zehn Jahren - mit automatischer Verlängerung - und nach zwölfmonatiger Kündigungsfrist eine fortdauernde Schutzwirkung von weiteren $z$ wanzig Jahren zu vereinbaren.

\section{Die Weltbank-Schiedsgerichtskonvention}

Unabhängig von ihrem Bestreben, möglichst zahlreiche Investitionsförderungsverträge abzuschließen, ist die Bundesrepublik Deutschland bereits 1966 dem „Weltbank-Übereinkommen zur Beilegung von Investitionsstreitigkeiten zwischen Staaten und Angehörigen anderer Staaten " beigetreten.

Dieses Übereinkommen stellt gegenüber dem allgemeinen Völkerrecht insofern einen Fortschritt dar, als es neben den Staaten auch natürlichen oder juristischen Einzelpersonen Zugang zu einer internationalen Schiedsgerichtsbarkeit eröffnet. Weiterhin schafft es im Gegensatz zu bilateralen Verträgen durch seine internationale Grundlage eine Einheitlichkeit aller Schiedsverfahren über Investitionsstreitigkeiten. Letztlich klärt das Übereinkommen aufgrund seiner ausführlichen Verfahrensordnungen auch verschiedene bislang in der internationalen Schiedsgerichtsbarkeit zweifelhafte Fragen.

Der materielle Inhalt des Weltbank-Übereinkommens kann hier nur in seinen Grundzügen dargestellt werden. Seine Regelungen und seine Wirkungen beruhen auf folgenden Grundlagen ${ }^{5}$ :

- „Vor der Vergleichskommission und dem Schiedsgericht sind (kapitalimportierende) Staaten und (private) Investoren parteifähig.

- Nach Einigung über die Zuständigkeit dieser Organe kann keine Partei die Durchführung des Verfahrens einseitig verhindern.

5 Vgl. Gerd Langer: Das Weltbank-Ưbereinkommen zur Beilegung von Investitionsstreitigkeiten. In: Außenwirtschaftsdienst des Betriebsberaters, Heft 7, Juli 1972, S. 322. 
- Haben sich die Parteien nicht über das anwendbare materielle Recht geeinigt, so verweist die Konvention auf das Recht des Gaststaates sowie die einschlägigen Regeln des Völkerrechts.

- Die Schiedssprüche sind bindend und wie innerstaatliche Urteile vollstreckbar."

Aufgrund des Übereinkommens wurde ein Internationales Zentrum am Sitz der Weltbank in Washington errichtet. Für die Behandlung von Streitfällen unterhält das Zentrum getrennte Gremien von Vermittlern und Schiedsrichtern, von denen die meisten von den Vertragsstaaten und einige von dem Vorsitzenden des Verwaltungsrates bestimmt werden. Im Rahmen der Konvention verhandelnde Parteien können die Mitglieder ihrer Vergleichskommissionen oder Schiedsgerichte aus diesen Gremien wählen, müssen es aber nicht.

Das Zentrum - je nach dem Antrag eine Vergleichskommission oder ein Schiedsgericht - ist zuständig, wenn beide Parteien nach den Bestimmungen des Übereinkommens parteifähig sind, sie sich vorab über die Zuständigkeit des Zentrums geeinigt haben, und der Streitfall seiner Rechtsnatur nach eine Investitionsstreitigkeit ist.

Das Zentrum dient ausschließlich der Beilegung von Streitigkeiten zwischen Staaten und Angehörigen anderer Staaten. Somit können nicht zwei Staaten als Parteien gegeneinander auftreten. Ebensowenig kann ein Angehöriger eines Vertragsstaates seinen eigenen Heimatstaat verklagen. Die Möglichkeit der Schlichtung ohne Einschaltung des Heimatstaates des Investors soll zudem dazu beitragen, die Streitfälle zu entpolitisieren.

Ist eine Vergleichs- oder Schiedsabrede getroffen worden, so kann der Heimatstaat des Investors zu dessen Gunsten nicht mehr den völkerrechtlichen Schutz ausüben. Das Gastland soll nicht gleichzeitig einem Verfahren vor dem Zentrum und Schritten des Heimatstaates des Investors ausgesetzt sein.

\section{Zur Bedeutung staatlicher Garantien}

Die Wirksamkeit der einzelnen Schutzmaßnahmen zugunsten von Direktinvestitionen in Entwicklungsländern hängt von verschiedenen Einflußgrößen ab, so zum Beispiel von der speziellen Ausgestaltung der Einzelmaßnahme, von der besonderen Situation des Investors - vor allem seinen Investitionsmotiven und alternativen Investitionsmöglichkeiten - sowie von dem gewählten Anlageland und dem dortigen Investitionsklima. Auch ist sicherlich mitentscheidend, was als Ziel der Schutzmaßnahmen gesehen wird: lediglich eine Absicherung oder eine allgemeine Erhöhung der deutschen Direktinvestitionen in Entwicklungsländern, eine Auswahl entwicklungspolitisch bedeutsamer Investitionen bzw. gar eine Lenkung des Kapitals in bestimmte Regionen oder Staaten.

Staatliche Garantien oder Versicherungen gegen (entschädigungslose) Enteignungen von Direktinvestitionen in Entwicklungsländern sind sicher als eines der wesentlichsten Förderungsinstrumente zu bezeichnen, da gerade das politische Risiko einer dortigen Kapitalanlage oft noch als besonders groß betrachtet wird. So versuchen viele Entwicklungsländer, durch entsprechende Zusagen in ihren Verfassungen, Investitionsgesetzen, Niederlassungsverträgen oder Investitionsförderungsverträgen oder durch Beitritt zur Weltbank-Schiedsgerichtskonvention einen Anreiz auf ausländische Investoren auszuüben. 
In einigen Staaten genügen diese Garantien dem Investor, in anderen jedoch nicht. Dies beweist der zusätzliche Abschluß von nationalen Investitionsversicherungen, der den Unternehmer zudem mit Kosten belastet. Allerdings kann er im Schadensfall dann auch einen unmittelbaren Ersatzanspruch geltend machen, während er in den anderen Fällen auf langwierige Verhandlungen angewiesen ist. Jedoch steht es dem Unternehmer frei, für welche Länder er seine Auslandsinvestitionen versichert. So deutet die regionale Verteilung der staatlichen Garantien für Kapitalanlagen auch auf die Größe des politischen Risikos hin, das die Investoren den einzelnen Staaten oder Regionen zuordnen.

In der Bundesrepublik Deutschland wurden bis zum 31. 1. 1972 insgesamt 985 Direktinvestitionen in Entwicklungsländern mit einem Garantiehöchstbetrag ( $\mathrm{Ka}$ pital- und Ertragsdeckung) von 1725 Mill. DM versichert, dies entsprach $22 \%$ aller investierten Mittel. Dabei war der Deckungsanteil in Afrika mit 40,2\% und in (Latein-) Amerika mit 30,4\% besonders hoch, während er in Asien mit 19,4\% fast dem Durchschnitt entsprach und in Europa mit 10\% sehr niedrig war (Tab. 2). Etwa 25\% aller Garantieanträge wurden genehmigt.

Tabelle 2:

Garantierte deutsche Direktinvestitionen in Entwicklungsländern

(Stand 31. 12. 1972)

\begin{tabular}{lccccc}
\hline & $\begin{array}{c}\text { Anzahl } \\
\text { der Länder }\end{array}$ & \multicolumn{2}{c}{$\begin{array}{c}\text { Investitionen } \\
\text { (Anträge) }\end{array}$} & \multicolumn{2}{c}{$\begin{array}{c}\text { Versicherte } \\
\text { Höchstbeträge }\end{array}$} \\
& Anzahl & $\%$ & in $\%$ & in Mill. DM \\
\hline Afrika & 30 & 325 & 33,0 & 40,2 & 693,5 \\
Amerika & 19 & 353 & 35,9 & 30,4 & 524,5 \\
Asien & 19 & 199 & 20,2 & 19,4 & 334,5 \\
Europa & 6 & 108 & 11,0 & 10,0 & 172,5 \\
\hline Summe & & 985 & 100,1 & 100,0 & 1725,0 \\
\hline
\end{tabular}

Quelle: Gerd Langer: Der Rechtsschutz und die Übernahme von Garantien zur Absicherung des politischen Risikos für Kapitalanlagen (Direktinvestitionen) in Entwicklungsländern. Beilage 21 zum Bundesanzeiger, Köln, Nr. 135 vom 24. 7. 1973, S. 6, sowie eigene Berechnungen.

\section{Die Anreizwirkung der Investitionsförderungsverträge}

Zwischen der regionalen Verteilung der Garantien und der der Investitionsförderungsverträge bestehen einerseits Parallelen, andererseits wurden aber in allen Regionen mit Ausnahme Europas mehr Direktinvestitionen versichert als in Länder mit Investitionsförderungsverträgen flossen. Daraus könnte gefolgert werden, „daß die Kapitalschutzabkommen aus der Sicht der Investoren wenig Schutz bieten und diese sich lieber auf die Garantieversprechen der Bundesregierung verlassen, zweitens aber, daß die Bundesregierung entsprechend der Vorschrift der Haushaltsgesetze bereitwilligst Garantien bei Kapitalschutzverträgen zusagt, darüber hinaus jedoch nur zögernd einwilligt ${ }^{6}$.

6 Günter Grosche: Deutsche Direktinvestitionen in Entwicklungsländern. Diss. Bochum 1971, S. 257. 
Die Bedeutung der Investitionsförderungsverträge liegt für die Bundesrepublik Deutschland zunächst darin, die wirtschaftliche Zusammenarbeit zu vertiefen, günstige Bedingungen für Kapitalanlagen zu schaffen und - durch deren Förderung und Schutz - die private wirtschaftliche Initiative $\mathrm{zu}$ beleben. Zugleich verringern die Verträge das Risiko der Bundesregierung, das sie bei der Gewährung von Garantien gegen politische Risiken bei Auslandsinvestitionen übernimmt.

Sofern die Verträge den Umfang der deutschen Direktinvestitionen in Entwicklungsländern positiv beeinflussen, tragen sie dazu bei, daß die Bundesrepublik dem von ihr anerkannten Ziel näher kommt, jährlich finanzielle Mittel in Höhe von einem Prozent des Bruttosozialprodukts in die Entwicklungsländer fließen zu lassen. Weiterhin erlauben es die Verträge der Bundesregierung, über die Anknüpfung wirtschaftlicher Kontakte $\mathrm{zu}$ engeren politischen Beziehungen $\mathrm{zu}$ gelangen.

Für die Entwicklungsländer liegt der Hauptgrund zum Abschluß von Investitionsförderungsverträgen in ihren Bemühen um mehr Kreditwürdigkeit, um eine größere Attraktivität für ausländische Investoren. Außerdem ist den Entwicklungsländern bewußt, daß bei vielen Industrieländern die Gewährung von staatlichen Investitionsgarantien - die oft ein wesentlicher Einflußfaktor sind - an das Vorhandensein von Investitionsklauseln oder -verträgen gebunden ist.

$\mathrm{Da}$ die Investitionsförderungsverträge nicht für alle Entwicklungsländer gelten, läßt sich - anders als bei den staatlichen Garantien - eventuell ein Zusammenhang zwischen dem Umfang der Direktinvestitionen und dem Bestehen solcher Verträge feststellen. Dazu ist einerseits zu prüfen ${ }^{7}$, inwieweit die regionale Struktur der Direktinvestitionen mit der Verteilung der Verträge auf die einzelnen Entwicklungsländer übereinstimmt. Andererseits ist $\mathrm{zu}$ ermitteln, ob sich aus der quantitativen Entwicklung der Direktinvestitionen in jedem einzelnen Vertragsstaat eine Relation zum Zeitpunkt der Unterzeichnung bzw. des Inkrafttretens des Vertrages ergibt.

Der Vergleich der Verteilung von Direktinvestitionen und Investitionsförderungsverträgen auf die einzelnen Erdteile zeigt völlig entgegengesetzte Schwerpunkte. 1972/73 lagen ca. $45 \%$ aller deutschen Direktinvestitionen (in Entwicklungsländern) in Lateinamerika, wo nur ein einziger Vertrag in Kraft ist, während auf die afrikanischen Entwicklungsländer mit $66 \%$ aller Verträge nur $19 \%$ der Direktinvestitionen entfielen. Dies zeigt bereits, daß von den Verträgen zumindest keine entscheidende Wirkung auf die Standortwahl bei Investitionen in Entwicklungsländern ausging.

Dies wird noch deutlicher, wenn man den Anteil der Vertragsstaaten an den deutschen Direktinvestitionen in Entwicklungsländern betrachtet. Ende 1972 lagen $14 \%$ und Ende 1973 nur 13\% dieser Investitionen in Staaten mit Investitionsförderungsverträgen. Demnach ist auf jeden Fall für die restlichen $86 \%$ bzw. $87 \%$ ein Vertrag nicht ausschlaggebend gewesen. Die Betrachtung der Verteilung in den einzelnen Erdteilen bringt nur ein geringfügig anderes Ergebnis.

Die Analyse der Entwicklung des Umfangs deutscher Direktinvestitionen in Entwicklungsländern mit Investitionsförderungsverträgen von 1963 bis 1973 zeigt,

7 Die folgenden Ausführungen basieren auf umfangreicheren Berechnungen des Verfassers, vgl. Heinrich Jüttner: Förderung und Schutz deutscher Direktinvestitionen in Entwicklungsländern. Baden-Baden 1975 , S. $290 \mathrm{ff}$. 
daß der Gesamtbetrag zwar laufend zunahm, anteilmäßig jedoch von 1966 an keine Steigerung mehr erfuhr, obwohl die Zahl der Vertragsstaaten sich seitdem von neun auf 32 erhöhte. Dieses Ergebnis läßt auf eine eher negative Investitionsentwicklung in den Vertragsstaaten schließen.

Die Untersuchung, ob die Entwicklung der deutschen Direktinvestitionen in den einzelnen Vertragsstaaten in einem zeitlichen Zusammenhang mit dem Inkrafttreten des Investitionsförderungsvertrages steht, führt zu dem Ergebnis, daß in zehn Staaten der Anteil an den deutschen Direktinvestitionen nach diesem Zeitpunkt etwa gleich blieb, in neun Staaten sogar sank, in vier Staaten kurzfristig anstieg, dann aber wieder zurückging und nur in neun Staaten relativ stetig zunahm bzw. auf ein höheres Niveau stieg. Allein dort könnte sich also die Frage nach einer Relation mit dem Investitionsförderungsvertrag erheben.

Zusammenfassend läßt sich sagen, daß aus der statistischen Analyse kein unmittelbarer Zusammenhang $\mathbf{z w i s c h e n ~ I n v e s t i t i o n s u m f a n g ~ u n d ~ I n v e s t i t i o n s f o ̈ r d e r u n g s - ~}$ vertrag abzuleiten ist, unabhängig davon ob der Investitionsbestand oder die jährlichen Neuinvestitionen verglichen werden, ob eine statische oder dynamische Betrachtung der Direktinvestitionen erfolgt und ob als Zeitpunkt die Unterzeichnung oder das Inkrafttreten des Investitionsförderungsvertrags herangezogen wird.

Mögliche Zusammenhänge lassen sich nur in wenigen Fällen erkennen, so daß die Anreizwirkung der Investitionsförderungsverträge auf deutsche Direktinvestitionen insgesamt als gering einzustufen ist. Das Bestehen oder Fehlen eines Investitionsförderungsvertrags wird bei der unternehmerischen Entscheidung über Direktinvestitionen in Entwicklungsländern zwar zur Kenntnis genommen, übt aber keine nachweisbare investitionserhöhende oder -lenkende Wirkung aus.

\section{Erfahrungen mit der Schutzwirkung}

Hieran anschließend erhebt sich die Frage, ob sich - anders als die Anreizwirkung - die Schutzwirkung der Investitionsförderungsverträge in der Praxis bewährt hat, ob die vertraglichen Garantien eingehalten wurden, ob es zu Investitionsstreitigkeiten gekommen ist und welche Erledigung diese fanden.

Laut Auskunft des Bundesministeriums für Wirtschaft vom 10. März 1976 hatte es bis zu diesem Zeitpunkt noch kein Schiedsverfahren nach dem Investitionsförderungsvertrag gegeben. Die vertraglichen Garantien sind demnach stets eingehalten worden, und in den zuständigen Bundesressorts war man sich einig, daß diese völkerrechtlichen Abmachungen zumindest erhebliche psychologische Barrieren errichteten, die auch ein Staat, der vielleicht aus innenpolitischen Gründen zur Enteignung neigt, nicht einfach ignorieren kann.

1971 hatte die Bundesregierung sogar ausdrücklich festgestellt, daß die Verträge von den Partnerstaaten korrekt eingehalten würden. Es seien auch keine Fälle bekannt, in denen Enteignungen, sofern sie erfolgten, in diskriminierender Weise gegen deutsche Investitionen gerichtet worden wären. Deutsche Kapitalanlagen befänden sich überwiegend in der verarbeitenden Industrie und würden von solchen Maßnahmen nicht unmittelbar berührt. Auch würden sie in vielen Fällen gemeinsam mit inländischen Investoren in Form von „joint ventures“ vorgenommen, womit die Bereitschaft bekundet werde, das Unternehmen in die nationale Wirtschaft einzugliedern ${ }^{8}$.

8 Vgl. Enteignungen in der Dritten Welt. In: Handelsblatt vom 30. August 1971. 
Seitens der deutschen Wirtschaft wurde ebenfalls darauf hingewiesen, daß es „erfreulicherweise" noch nicht notwendig war, die Frage nach dem "Wert" in Kraft befindlicher Verträge im Falle von schwerwiegenden Eingriffen zu beantworten. „Nur in wenigen Fällen mußte sich die Bundesregierung auf den Vertrag stützen, um Maßnahmen des Entwicklungslandes zu korrigieren9."Seitens der Schweiz, die acht Investitionsschutzverträge und sechzehn Investitionsschutzklauseln vereinbart hat, ist (bis September 1974) ebenfalls noch kein Schiedsgerichtsverfahren eingeleitet worden.

Bei einer Unternehmensbefragung des Verfassers über Investitionsförderungsverträge wurde erfragt, ob die Investoren bereits Erfahrungen mit der Schutzwirkung der Verträge gemacht hatten und ob ihnen gegebenenfalls der Vertrag zur Durchsetzung ihrer Ansprüche verholfen hatte. In fast allen Antworten wurde diese Fragen verneint, in wenigen Fällen hatte man sich auf Bestimmungen des Vertrages erfolgreich berufen, in einem Fall war die Angelegenheit nicht zur Zufriedenheit des Investors erledigt worden. $\mathrm{Zu}$ einem Schiedsgerichtsverfahren war es bei keiner Investition gekommen.

Anders stellt sich die Situation bezüglich des Weltbank-Übereinkommens zur Beilegung von Investitionsstreitigkeiten dar, das allgemein als eine erfolgreiche Lösung anerkannt wird und dessen Musterklauseln schon Bestandteil zahlreicher Niederlassungs- und Investitionsschutzverträge sowie sogar nationaler Investitionsgesetze geworden sind, auch wenn das Zentrum mangels Meldepflicht keine genauen Zahlen angeben kann.

Eine erste Investitionsstreitigkeit wurde dem Zentrum am 27. Dezember 1971 zur Beilegung unterbreitet, beteiligt waren die Holiday Inns, S. A. und die Occidental Petroleum Corporation auf der einen, die Regierung von Marokko auf der anderen Seite. Bis Mitte 1975 war noch keine Entscheidung ergangen; diese darf zudem nur mit Zustimmung der Parteien veröffentlicht werden. Im ersten Halbjahr 1974 wurden dem Zentrum vier weitere Investitionsstreitigkeiten vorgelegt. Beteiligt waren drei US-amerikanische Firmen und ein italienisches Unternehmen sowie die Regierungen von Jamaika (dreimal) und der Elfenbeinküste ${ }^{10}$.

Die Betonung der prozessualen Seite in der Weltbank-Konvention wird zwar einerseits als wichtige Voraussetzung für die weite Anerkennung des Übereinkommens bezeichnet, andererseits in Wirtschaftskreisen aber auch als Nachteil dieser Konvention betrachtet. So wird bemängelt, daß keine Regeln des Wohlverhaltens, etwa für die Behandlung des ausländischen Eigentums oder für die Beziehungen zwischen ausländischen Investoren und den Gastländern aufgestellt seien und daß die Konvention ein „Schiedsgerichtsverfahren nur auf freiwilliger Basis vorsieht und vor allem, daß sie keinerlei materielle Schutzregeln als Richtschnur für die Entschedung der Schiedsinstanzen enthält. Das heißt, wenn ein Schadensfall eintritt . . ., so ist es selbst einem der Konvention beigetretenen Land freigestellt $\mathrm{zu}$ entscheiden, ob es diesen Fall dem Schiedsgericht unterwirft oder nicht ${ }^{11}$."

Letztlich bleibt auch die Frage nach der Durchsetzbarkeit der Schiedsgerichtsentscheidungen offen. Das Abkommen stellt zwar fest, daß die Schiedssprüche bindend sind, keiner Berufung unterliegen, von jeder Partei genau zu befolgen sind

9 Paul Krebs in einem Referat auf der Sitzung der Arbeitsgemeinschaft Entwicklungsländer am 21. Nov. 1968 in Frankfurt.

10 Vgl. International Centre for Settlement of Investment Disputes (Hrsg.): Eighth Annual Report 1973/74. Washington 1974, S. 4.

11 Hermann-Josef Abs: Die rechtliche Problematik privater Auslandsinvestitionen. Karlsruhe 1969, S. 15. 
und wie die Urteile nationaler Gerichte zu vollstrecken sind, doch ist dies keine zwingende Garantie gegen willkürliche staatliche Eingriffe. Im Fall eines Verstoßes rechnet die Weltbank offensichtlich einerseits mit einem solidarischen Verhalten aller Vertragsstaaten, behält sich andererseits aber auch vor, die Gewährung von Krediten an einen vertragsbrüchigen Staat auszusetzen und ihm dadurch ausdrücklich die internationale Kreditwürdigkeit abzusprechen.

\section{Investitionsschutz und -förderung in der Unternehmenspolitik}

Um die Bedeutung staatlicher Schutzmaßnahmen für Direktinvestitionen in Entwicklungsländern im Rahmen der unternehmerischen Entscheidung beurteilen zu können, müssen zunächst die Investitionsmotive analysiert werden. Dabei empfiehlt es sich, auf Wirtschaftsbefragungen über die Investitionsmotive bei Auslandsengagements deutscher Unternehmen in Entwicklungsländern zurückzugreifen.

Wie die Erhebung des Verfassers aus den Jahren 1973/7412 (in Tabelle 3) zeigt, kommt den Motiven "Sicherung und Ausbau eines bisherigen Marktes" und „Ausdehnung der Auslandsaktivität auf neue Märkte“ eine klare Priorität zu. Beide Motive erreichen jeweils etwa ein Viertel sämtlicher von den Unternehmen vergebenen Nennungen und werden von rund $90 \%$ aller Firmen angeführt. Selbst unter Berücksichtigung der Tatsache, daß mögliche Antworten vorgegeben waren und die den Fragebogen ausfüllenden Personen eher diese Antworten ankreuzen werden als zusätzliche hinzufügen, zeigt die Erhebung klar die Dominanz des Absatz- bzw. Umsatzdenkens.

Auch das an dritter Stelle liegende „Zwangsmotiv“ der „Umgehung von Handelsund Exporthindernissen" ist noch unter die Überlegungen zur Marktsicherung einzuordnen. Die in der Offentlichkeit als vorrangig vermuteten Motive der Ausnutzung "niedriger Preise für Roh- und Betriebsstoffe und niedriger Löhne“ sowie die "Erwartung einer hohen Rendite“ folgen erst auf Rang vier und fünf mit nur $10 \%$ bzw. $8 \%$ aller Nennungen. Sie werden überhaupt nur von der Hälfte aller Firmen angeführt ${ }^{13}$.

Weiterhin macht die Untersuchung deutlich, daß staatlichen Förderungsmaßnahmen nur eine nachrangige Bedeutung bei der Investitionsentscheidung zukommt. Hier zeichnet sich ab, daß die staatliche Förderung zwar in die Investitionsüberlegungen einbezogen wird, jedoch keineswegs ausschlaggebend ist. Keine Auslandsinvestition wird allein wegen staatlicher Förderungseinrichtungen durchgeführt, lediglich die Entscheidung für oder gegen einen bestimmten Standort kann durch staatliche Investitionsförderung beeinflußt werden.

Ausgehend von dieser recht zurückhaltenden Bewertung der staatlichen Investitionsförderungsmaßnahmen ist jetzt zu untersuchen, welches Gewicht den einzelnen Instrumenten seitens der Investoren zugeordnet wird. Auch hier kann wieder auf die erwähnte Unternehmensbefragung des Verfassers zurückgegriffen werden (Tabelle 4). Dabei war die Frage nach der Bedeutung der Förderungsmaßnahmen

\footnotetext{
12 Heinrich Jüttner, a. a. O., S. $99 \mathrm{ff}$

$13 \mathrm{Zu}$ vergleichbaren Ergebnissen war in den Jahren 1968/69 eine Erhebung des HWWA-Institutes in Hamburg gekommen, vgl. Karl Fasbender und Wolfgang Menck: Probleme von Direktinvestitionen in Entwicklungsländern und Möglichkeiten ihrer rechtlichen Absicherung. In: Verfassung und Recht in Übersee, 2. Jg., 1971, Nr. 2, S. 189.

14 Erhebungen des Verfassers, vgl. Heinrich Jüttner, a. a. O., S. $271 \mathrm{ff}$.
} 
für die Investitionsentscheidung in drei Teile gegliedert: Förderungsmaßnahmen der Bundesrepublik Deutschland, der Entwicklungsländer und auf internationaler Ebene.

In der Skala der deutschen staatlichen Förderungsmaßnahmen nimmt das Entwicklungshilfe-Steuergesetz mit 30,0\% der Nennungen den ersten Rang ein, gefolgt von den Bundesgarantien gegen politische Risiken mit 23,6\%. Weitere Maßnahmen steuerlicher Art wie Doppelbesteuerungsabkommen stehen auf dem dritten Rang $(18,2 \%)$, während die Darlehen und Beteiligungen der Deutschen Entwicklungsgesellschaft $11,8 \%$ der Nennungen erhalten. An fünfter Stelle steht die andere Maßnahme zur Risikominderung (Investitionsförderungsverträge) mit 8,6\%. Dagegen bekamen die Finanzierungshilfen aus ERP-Mitteln oder nach dem Kriegsfolgengesetz nur 5,5\%. Zuschüsse für die Ausbildung von Fachkräften aus Entwicklungsländern erreichen lediglich $1,8 \%$ der Nennungen, und den Informationshilfen und Zuschüssen zu Investitionsstudien wird fast keine Bedeutung bei der Investitionsentscheidung zuerkannt $(0,5 \%)$.

Tabelle 3:

Entscheidungsfaktoren bei deutschen Direktinvestitionen in Entwicklungsländern

\begin{tabular}{|c|c|c|c|c|}
\hline \multirow{3}{*}{ Faktoren } & \multicolumn{4}{|c|}{ Antworten } \\
\hline & \multicolumn{2}{|c|}{ Firmen $^{1}$ ) } & \multicolumn{2}{|c|}{ Nennungen ${ }^{2}$ ) } \\
\hline & Anzahl & Prozent & Anzahl & Prozent \\
\hline Sicherung und Ausbau eines & & & & \\
\hline bisherigen Marktes & 42 & 91,3 & 107 & 26,8 \\
\hline $\begin{array}{l}\text { Ausdehnung der Auslands- } \\
\text { aktivität auf neue Märkte }\end{array}$ & 41 & 89,1 & 90 & 22,6 \\
\hline $\begin{array}{l}\text { Umgehung von Handels- bzw. } \\
\text { Exporthindernissen }\end{array}$ & 31 & 67,4 & 61 & 15,3 \\
\hline $\begin{array}{l}\text { Niedrige Preise für Roh- und } \\
\text { Betriebsstoffe, niedrige Löhne }\end{array}$ & 25 & 54,4 & 41 & 10,3 \\
\hline $\begin{array}{l}\text { Erwartung einer hohen } \\
\text { Rendite }\end{array}$ & 22 & 47,8 & 33 & 8,3 \\
\hline $\begin{array}{l}\text { Förderungsmaßnahmen für } \\
\text { Auslandsinvestitionen }\end{array}$ & 24 & 52,2 & 32 & 8,0 \\
\hline $\begin{array}{l}\text { Entwicklungspolitische und } \\
\text { volkswirtschaftliche Ziele }\end{array}$ & 12 & 26,1 & 17 & 4,2 \\
\hline $\begin{array}{l}\text { Sicherung und Erweiterung der } \\
\text { Rohstoffbasis }\end{array}$ & 7 & 15,2 & 17 & 4,2 \\
\hline $\begin{array}{l}\text { Beteiligungsübernahme bei } \\
\text { Anlagenerrichtung }\end{array}$ & 1 & 2,2 & 1 & 0,3 \\
\hline & & & 399 & 100,0 \\
\hline
\end{tabular}

Q u elle : Eigene Erhebungen

1) Mehrfachantworten waren möglich.

2) Jede Firma konnte jeden Faktor je nach Bedeutung mit 0 bis 3 Kreuzen bewerten. 
Tabelle 4:

Bedeutung der Investitionsförderungsmaßnahmen für die Investitionsentscheidung a) Förderungsmaßnahmen der BRD Firmen Nennungen*)

\begin{tabular}{|c|c|c|c|c|}
\hline 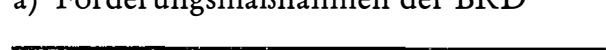 & Anzahl & Prozent & Anzahl & Prozent \\
\hline Entwicklungshilfe-Steuergesetz & 39 & 84,8 & 66 & 30,0 \\
\hline Bundesgarantien gegen politische Risiken & 29 & 63,0 & 52 & 23,6 \\
\hline $\begin{array}{l}\text { Doppelbesteuerungsabkommen und ein- } \\
\text { seitige steuerliche Erleichterungen }\end{array}$ & 27 & 58,7 & 40 & 18,2 \\
\hline Beteiligungen und Darlehen der DEG & 17 & 37,0 & 26 & 11,8 \\
\hline Investitionsförderungsverträge & 17 & 37,0 & 19 & 8,6 \\
\hline $\begin{array}{l}\text { Finanzierungshilfen aus ERP-Mitteln } \\
\text { oder nach dem Kriegsfolgengesetz }\end{array}$ & 8 & 17,4 & 12 & 5,5 \\
\hline $\begin{array}{l}\text { Zuschüsse für die Ausbildung einheimi- } \\
\text { scher Fachkräfte }\end{array}$ & 3 & 6,5 & 4 & 1,8 \\
\hline $\begin{array}{l}\text { Informationshilfen und Zuschüsse zu } \\
\text { Vorstudien }\end{array}$ & 1 & 2,2 & 1 & 0,5 \\
\hline
\end{tabular}

b) Internationale Förderungsmaßnahmen

\begin{tabular}{lrrrr}
\hline $\begin{array}{l}\text { Beteiligungen und Darlehen der Inter- } \\
\text { national Finance Corporation (IFC) }\end{array}$ & 5 & 10,9 & 9 & 75,0 \\
\begin{tabular}{l} 
Weltbank-Schiedsgerichtskonvention \\
\hline
\end{tabular} & 3 & 6,5 & 3 & 25,0 \\
\hline
\end{tabular}

c) Förderungsmaßnahmen der Entwicklungsländer

\begin{tabular}{|c|c|c|c|c|}
\hline Transfergarantien für Erlöse, Erträge & 40 & 87,0 & 88 & 28,7 \\
\hline Eigentumsgarantien & 34 & 73,9 & 68 & 22,1 \\
\hline Steuerbefreiungen und -erleichterungen & 31 & 67,4 & 52 & 16,9 \\
\hline $\begin{array}{l}\text { Einfuhrbegünstigungen (Zollfreiheit oder } \\
\text {-nachlaß, Devisenzuteilung etc.) }\end{array}$ & 30 & 65,2 & 49 & 16,0 \\
\hline $\begin{array}{l}\text { Finanzierungshilfen (Kredite, Zuschüsse, } \\
\text { Bereitstellung von Boden etc.) }\end{array}$ & 28 & 60,9 & 34 & 11,1 \\
\hline \multirow[t]{2}{*}{ Infrastrukturmaßnahmen } & 11 & 23,9 & 16 & 5,2 \\
\hline & & & 307 & 100,0 \\
\hline
\end{tabular}

\begin{tabular}{lll}
\hline An der Erhebung beteiligte Unternehmen & 46 & 100,0
\end{tabular}

Qu elle : Eigene Erhebungen

*) Die Unternehmen konnten jede Förderungsmaßnahme je nach Bedeutung mit 0 bis 3 Kreuzen kennzeichnen. 
Wenn demnach die Bundesgarantien für Kapitalanlagen im Ausland fast ein Viertel aller Nennungen erzielen und von zwei Dritteln aller Firmen angeführt werden, so unterstreicht dies klar die Bedeutung einer Sicherheitsgewährung für Direktinvestitionen. Ein unmittelbarer Einfluß auf die Investitionsentscheidung muß dieser Förderungsmaßnahme allein schon deshalb zukommen, weil der Investor selbst aufgrund seiner Einschätzungen des politischen Risikos festlegt, ob er sich um eine Garantie bemüht oder nicht. Zudem kann der Unternehmer — wie erwähnt - aus diesen Garantien einen direkten Ersatzanspruch ableiten, während dies bei den Investitionsförderungsverträgen und häufig auch bei den Garantieerklärungen der Entwicklungsländer nicht möglich ist. Daher werden die Investitionsförderungsverträge auch erheblich niedriger eingestuft und erhalten nur ein Drittel der Nennungen, die auf die Bundesgarantien entfielen.

Investitionsförderungsmaßnahmen auf internationaler Ebene werden von den deutschen Unternehmen als unbedeutend eingordnet. Nur wenige Investoren sprechen ihnen überhaupt eine Relevanz zu. Im Falle der IFC-Darlehen und -Beteiligungen mag gelten, daß die IFC bzw. ihre Tätigkeit nur wenigen deutschen Firmen ein Begriff ist. So erklärt sich auch, warum vor allem Großunternehmen Partnerschaften mit der IFC eingegangen sind. Die Weltbank-Schiedsgerichtskonvention dürfte ebenfalls weitgehend unbekannt und daher als unwesentlich beurteilt worden sein. Sie trat erst im Jahre 1966 in Kraft und ist bislang noch nicht mit besonderen Aktionen hervorgetreten.

Unter den Förderungsmaßnahmen der Entwicklungsländer liegen wiederum risikopolitische Maßnahmen, nämlich staatliche Garantien für den uneingeschränkten Transfer von Erträgen, Erlösen ect. und gegen Enteignungen bzw. entschädigungslose Enteignungen mit zusammen $51 \%$ der Nennungen an der Spitze der Rangfolge. Dies weist erneut auf die Bedeutung hin, die Sicherheitsüberlegungen gerade bei der Entscheidung über Direktinvestitionen in Entwicklungsländern spielen. Außerdem zeigt dieses Ergebnis, daß die Unternehmen Investitionsgesetzgebung und Wirtschaftsrecht der Entwicklungsländer beobachten und ihnen eine - wenn auch nur relative - Bedeutung beimessen.

\section{Investitionsförderungsverträge aus unternehmerischer Sicht}

Als spezielle Maßnahmen, die von Industrie- und Entwicklungsland gemeinsam zur Förderung und zum Schutz von ausländischen Direktinvestitionen ergriffen werden, sollen die Investitionsförderungsverträge aus der Sicht der Unternehmen näher beleuchtet werden. Dabei ist davon auszugehen, daß die Einstufung der Investitionsförderungsverträge innerhalb des Förderungsinstrumentariums einerseits und die Nachrangigkeit solcher staatlicher Förderungsmaßnahmen bei der Investitionsentscheidung andererseits bereits klargemacht haben, daß die Wirksamkeit dieser Verträge recht gering einzuschätzen ist. Allein unter diesem Aspekt sind daher die folgenden Detailbetrachtungen ${ }^{14} \mathrm{zu}$ sehen, ihre Aussagekraft wird durch die allgemeine Bedeutung der Investitionsförderungsverträge relativiert.

Die Frage nach der Eignung des Investitionsförderungsvertrages als Anreiz- oder Schutzmaßnahme brachte das zu erwartende Ergebnis, daß die Schutzwirkung des Vertrages höher eingestuft wird als seine Förderungswirkung. Zusammengefaßt sehen $90 \%$ aller Unternehmen, die sich hierzu eine Meinung gebildet haben, in 
den Verträgen einen mehr oder weniger starken Schutz für ihre ausländischen Kapitalanlagen. Eine Anreizwirkung sprechen dagegen nur knapp $80 \%$ dieser Unternehmen den Verträgen zu. Absolut gesehen ist dies jedoch überraschend viel und wahrscheinlich darin begründet, daß die Wirkung der Investitionsförderungsverträge hier losgelöst und nicht im Zusammenhang mit anderen Investitionsmotiven oder Förderungsmaßnahmen erfragt wurde.

Den unmittelbaren Zusammenhang zwischen dem Fehlen bzw. Bestehen eines Investitionsförderungsvertrages und der Investitionsentscheidung klärte eine weitere Frage, in der um die Zahl der Investitionsfälle gebeten wurde, in denen diese Verträge eine Bedeutung gehabt hatten. Hier entspricht das Ergbnis schon eher der tatsächlichen Effizienz der Verträge, da sie in jeweils einem Drittel aller Fälle ohne Bedeutung geblieben waren bzw. nur als Voraussetzung für die Übernahme einer Garantie gegen politische Risiken eine Relevanz erhielten. Bei 11 von insgesamt 88 Investitionsvorhaben war das Bestehen oder Fehlen eines Investitionsförderungsvertrages von wesentlicher und in 16 Fällen noch von geringer Bedeutung. Bei zwei Investitionsentscheidungen war dem Bestehen eines Vertrages sogar „ausschlaggebende Bedeutung " zugesprochen worden.

Recht unterschiedlich wurden die einzelnen Bestimmungen eines Investitionsförderungsvertrags von den befragten Unternehmen bewertet. An der Spitze standen mit $22 \%$ bzw. mit $20 \%$ der Nennungen der Schutz vor ungerechtfertigter Enteignung und die Entschädigungsgarantie, also wiederum Aspekte des Vermögensschutzes. Dem folgten Inländerbehandlung (15\%), Schutzfortdauer nach Vertragsablauf $(11 \%)$, Voraussetzung für Bundesgarantien $(10 \%)$, Meistbegünstigungsklausel $(8 \%)$, internationale Schiedsgerichtsbarkeit $(7 \%)$ und Besserungsklausel $(5 \%)$.

Innerhalb der verschiedenen Arten von Garantien wurde eine entsprechende internationale Vereinbarung von $56 \%$ der Unternehmen höher, von $42 \%$ etwa gleich und nur von $2 \%$ niedriger eingestuft als ein Investitionsförderungsvertrag. Inhaltlich gleiche Garantien eines Entwicklungslandes erhielten dagegen eine unterschiedliche Bewertung. Für 41,5\% der Investoren hatten sie eine höhere oder gar viel höhere Bedeutung; und jeweils $29 \%$ Investoren schätzten sie etwa gleich oder niedriger ein als einen Investitionsförderungsvertrag.

Zusammenfassend ist hierzu festzuhalten, daß den Investitionsförderungsverträgen in dieser Erhebung ein wider Erwarten großer Anteil an der unternehmerischen Entscheidung zugestanden wurde, nachdem dieses Förderungsinstrument zuvor in größerem Zusammenhang nur als „zweitklassig“ eingestuft worden war. Die Verträge werden demnach wohl doch in fast jede Investitionsentscheidung einbezogen und verlieren erst in der Gegenüberstellung mit anderen Faktoren an Bedeutung. 


\title{
Legal Protection of Foreign Investments in Developing Countries
}

\author{
By HeINRICH JüTTNER
}

The demand of the developing countries for full souvereignty over their national ressources und their economic activities takes a great role in the discussion on a new international economic order. Therefore the question of legal protection of foreign investments in the Third World is of great importance too. It is well known indeed, that developing countries as well as industrial countries are interested in foreign investments in developing countries. So they offer a broad catalogue of measures for the legal security of those investments.

The Federal Republic of Germany for example has national guarantees against political risks and bilateral investment protection agreements, until now with 44 developing countries. In the multilateral level there is the "Convention on the Settlement of Investment Disputes between States and Nationals of other States", administered by the World Bank. Against that the "Multilateral Investment Insurance", proposed by the World Bank, and the "Convention on the Protection of Foreign Property", prepared by the OECD, have not yet been realized. The developing countries offer different forms of transfer and property guarantees in their investment laws and arrangements.

Governmental guarantees or insurances against expropriation of foreign investments in developing countries are one of the most important investment incentives. Sometimes the investors are satisfied with the relevant promises of the developing countries; in many cases, however, they effect additional national insurances, because they get a direct claim for compensation in this way. In the Federal Republic of Germany more than $20 \%$ of all foreign investments in the Third World are protected by governmental guarantees against political risks.

The Investment Protection Agreements are classified less important by the investors, because the enforcement of the promises und guarantees in these treaties is depending from the cooperation climate and the legal system in the developing country. Inquiries have come to the result, that there is no incitement effect of these agreements regarding additional foreign investments or investments in certain developing countries. On the other hand their protection effect is positive and generally recognized.

The International Convention for the Settlement of Investment Disputes is regarded as a successful way to strengthen the legal security of foreign investments. So it is already part of many investment treaties, protection agreements and laws. First investment disputes are submit ted to the responsible centre, which has been established by the World Bank. German investors indeed prefere furthermore the governmental German investment guarantees.

\section{Dualism in Criminal Law and Court System in Nigeria}

\section{By BARbara Huber}

There are two criminal codes - with minor modifications in certain states which apply in contemporary Nigeria: The Nigerian Criminal Code of 1916 (NCC) 